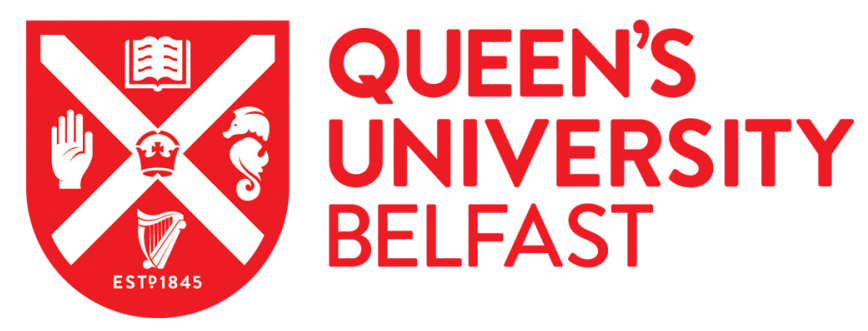

\title{
Association between oral health status and future dietary intake and diet quality in older men: the PRIME study
}

Logan, D., McEvoy, C. T., McKenna, G., Kee, F., Linden, G., \& Woodside, J. V. (2020). Association between oral health status and future dietary intake and diet quality in older men: the PRIME study. Journal of Dentistry, 92, 103265. https://doi.org/10.1016/j.jdent.2019.103265

Published in:

Journal of Dentistry

Document Version:

Peer reviewed version

Queen's University Belfast - Research Portal:

Link to publication record in Queen's University Belfast Research Portal

Publisher rights

Copyright 2019 Elsevier

This manuscript is distributed under a Creative Commons Attribution-NonCommercial-NoDerivs License

(https://creativecommons.org/licenses/by-nc-nd/4.0/), which permits distribution and reproduction for non-commercial purposes, provided the author and source are cited.

\section{General rights}

Copyright for the publications made accessible via the Queen's University Belfast Research Portal is retained by the author(s) and / or other copyright owners and it is a condition of accessing these publications that users recognise and abide by the legal requirements associated with these rights.

Take down policy

The Research Portal is Queen's institutional repository that provides access to Queen's research output. Every effort has been made to ensure that content in the Research Portal does not infringe any person's rights, or applicable UK laws. If you discover content in the Research Portal that you believe breaches copyright or violates any law, please contact openaccess@qub.ac.uk. 


\section{Abstract}

Title: Association between oral health status and future dietary intake and diet quality in older men: the PRIME study

Objectives: This study investigated whether oral health status, defined as number of natural teeth and subsequent prosthodontic rehabilitation, was associated with future dietary intake and diet quality in older adults in The Prospective Epidemiological Study of Myocardial Infarction (PRIME).

Methods: PRIME was originally established to explore cardiovascular risk factors in 50-59 year old men in Northern Ireland (1991-1994). A rescreening phase assessed oral health (2001-2004), while diet was assessed in 2015. Diet quality was characterised by the Dietary Diversity Score and Mediterranean Diet Score. In the current analysis, associations between oral health status, dietary intake and quality were assessed using regression models in 1,096 participants.

Results: Amongst study participants, the overall mean number of teeth was $18.5,51.5 \%$ had $\geq 21$ natural teeth and $49.6 \%$ wore dentures. Oral health status was categorised into five groups: 21-28 teeth with $(n=111)$ and without $(n=453)$ dentures, 1-20 teeth with $(n=354)$ and without $(n=99)$ dentures and edentate with dentures $(n=79)$. After full adjustment, men with $\geq 21$ teeth and dentures had a higher future intake of fruit, vegetables, and nuts, and diet quality scores, compared to those with $<21$ teeth with dentures. Edentate men with dentures were less likely to achieve the future fruit dietary recommendation.

Conclusions: Having $\geq 21$ natural remaining teeth positively affected the future intake of fruit, vegetables, and nuts, as well as diet quality. Dentures may be beneficial in men with $\geq 21$ natural remaining teeth, as they were associated with an increased future intake of fruit, vegetables, and nuts and better diet quality.

Clinical Significance: Oral health status is associated with dietary intake, after an average time period of 13 years, with those with a larger number of natural teeth having a better diet quality. Further research is required to investigate this relationship in larger, diverse populations with more detailed dietary assessment. 


\section{$\underline{\text { Introduction }}$}

Globally, the number of older adults is increasing and is projected to reach over 2.1 billion by 2050 [1]. Consequently, this may place additional demands on healthcare services, as the incidence and prevalence of age-related chronic diseases are also projected to increase, including: cardiovascular disease (CVD), stroke, chronic respiratory diseases and diabetes [2]. A healthy diet may play a role in reducing the risk of these chronic conditions. For example, the Mediterranean Diet (MD) is a dietary pattern mainly consumed in areas within the Mediterranean region and is associated with a reduced risk of CVD [3]. It consists of a high intake of fish, olive oil, non-starchy vegetables, legumes, wholegrains (cereals), fruits, and nuts, as well as a lower intake of dairy products, red and processed meat and a moderate intake of wine [4]. Dietary recommendations vary worldwide and are adapted to suit a country's specific needs, including food availability and cultural acceptability of specific foods [5]. The United Kingdom (UK) dietary guidelines recommend a plant-based dietary pattern for optimal health; individuals are advised to consume at least 2 portions of fruit and 3 portions of vegetables a day, a maximum of 1 portion $(70 \mathrm{~g})$ of red and processed meat a day [6] and at least 1 portion (140g) of oily fish a week [7]. The consumption of wholegrain options, some nuts and limited alcohol intake are also encouraged. Diet quality can be used to reflect compliance to dietary recommendations [8]. Globally, many older people have suboptimal intakes of fruit, vegetables, nuts, wholegrains and fish [9].

Dietary intake and diet quality in older adults is affected by various factors, such as, nutritional knowledge, physical health, loss of appetite, living circumstances and access to resources including transport [10]. Oral health status may also be an important factor influencing diet in terms of food choice and eating behaviour [10]. Changes in older adults' oral health have been observed over time in the United States (US) and UK, specifically a decline in the level of edentulism and increased tooth retention, and this has resulted in a partially dentate older population. In the US National Health and Nutrition Examination Survey (NHANES), the number of edentate $\geq 65$ year olds has decreased from 34\% in 1988-1994 to $27 \%$ in 1999-2004 [11]. The mean number of teeth has increased from 17.9 in 1988-1994 to 18.9 in 1999-2004 in the same population group [11]. From 2011-2016, the number of edentate older adults was estimated to be around $9 \%$ in those with high school education, and the mean number of teeth ranged from 16 to 22 across socio-demographic characteristics [12]. Similarly, the latest UK Adult Dental Health Survey, which is carried out every ten years, found that the number of edentate individuals aged $65-74$ years reduced from $78 \%$ in 1978 to $15 \%$ in 2009 and to $33 \%$ in 75+ year olds [13]. Those aged over 55 years old with 21 or more natural teeth, increased from $30 \%$ in 1978 to $63 \%$ in 2009 [13]. The mean number of teeth also increased, from 16.0 in 1978 to 21.2 in 2009 [13]. Despite improvements in older adults' oral health as discussed above, tooth loss still remains a global problem at present in older adults which has resulted in a partially dentate population [14]. For the purposes of this paper, oral health status was categorised according to the number of 
remaining natural teeth and, subsequent, prosthodontic rehabilitation. Edentulism was defined according to the Journal of Prosthodontic terms, as the state of being edentulous, i.e. without the presence of natural teeth [15]. Tooth loss is, therefore, considered an effective marker of oral health in a population [14]. It is recognised that excellent oral health consists of five key criteria, one of which is having 21 or more natural teeth [13]. This cut-off for 21 or more teeth has been proposed as the minimum number above which chewing ability should not be affected [13].

$\underline{\text { Recent evidence has suggested that older adults with less teeth or being edentate, may lead to the }}$ avoidance of foods perceived as difficult to eat including: raw vegetables, apples and nuts [16].Having a greater number of natural teeth has been associated with better nutritional status [17] and a higher intake of some foods including, fruit and vegetables and also nutrients, particularly fibre and vitamins [18]. Severe tooth loss has been associated with a lower overall diet quality in older adults and, subsequently, a greater number of teeth is associated with a higher diet quality [19-24], while having 21 or more natural teeth did not contribute substantially to a higher diet quality in older adults in the US [25]. Inconsistency in findings may be due to the studies being conducted in different countries and the use of different diet quality indices. The majority of this evidence is derived from cross-sectional studies, therefore it is difficult to discern the temporal nature of the relationship between oral health and diet, and it is possible that poor diet quality leads to tooth loss. A systematic review of eight longitudinal studies revealed contradictory results in relation to tooth loss, dietary intake and nutritional status in community-dwelling older adults in Australia, US, Brazil and Japan [26]. Some studies found that greater tooth loss may be associated with a reduced food intake of fruit and vegetables, and reduced nutrient intake of dietary fibre, and increased cholesterol, but overall findings were inconclusive [26]. The review concluded that amongst the few studies identified, results were inconsistent and there was no strong evidence regarding the effect of tooth loss on diet and nutrition [26]. However, in the quality assessment, seven of these studies were considered of poor quality and one was of fair quality [26]. Therefore, further high quality longitudinal studies are required in other countries to enhance the generalisability of the results and help inform policy and practice [26].

Evidence linking oral health status with dietary intake and quality in older adults is inconsistent and limited by the small numbers of prospective studies to date. The relationship between oral health status with both dietary intake and diet quality, has not been investigated together in this population of community-dwelling older adults. To our knowledge, the impact of oral health status on adherence to the MD has not been previously explored. Therefore, the aim of this study was to investigate whether oral health status, defined as the number of natural teeth and subsequent prosthodontic rehabilitation, was associated with dietary intake and diet quality in older men, after an average time period of 13 years. It was hypothesised that older men with fewer natural teeth and dentures would have a reduced 
dietary intake and diet quality, compared to those with a greater number of natural teeth and no dentures.

\section{Methods}

\section{Study Population}

The Prospective Epidemiological Study of Myocardial Infarction (PRIME) is an ongoing cohort study. PRIME was originally established to identify reasons for high levels of heart disease in men, by examining the association between CVD and a series of identified risk factors. Full sampling procedures and study design are described elsewhere [27]. In brief, between 1991 and 1994, men $(\mathrm{n}=2,745)$ were recruited from Belfast in Northern Ireland (NI) [27]. The sampling framework was chosen to reflect the social class structure of the background populations, based at different employment groups and health screening centres [27]. The men were followed up by means of an annual postal questionnaire from year 1-10 (1995-2005) and then again at year 25 (2015). They also attended a follow-up examination between 2001 and 2004, and during this time 1,558 men had their oral health assessed (mean age, 63.5 \pm 2.9 years). By the follow up stage in 2015, 1,029 men had died. The current study sample comprised 1,096 older men who had their oral health assessed between 2001 and 2004 and reported their dietary intake in 2015. Ethical approval was obtained from the appropriate Research Ethics Committee and, written consent was obtained from the men at the baseline examination and confirmed prior to the follow-up assessments.

\section{Data Collection}

Baseline (1991-1994)

Men self-completed standardised questionnaires to assess demographic and socio-economic background [27]. Material conditions acted as a proxy measure for socio-economic status (SES), where participants were categorised as low, intermediate or high [28]. Additional questionnaires were completed with the interviewer at their clinic visit, including: dietary intake, alcohol consumption using a weekly diary, and smoking status categorised as a current smoker, past smoker or never smoked. Medical information included whether participants had ever had high cholesterol or hypertension. Diabetes status was determined as the current intake of hypoglycaemic treatment or insulin use. Fasting venous blood samples were collected, processed within 4 hours and aliquoted for immediate analysis or low temperature storage [27]. Samples were analysed for various biomarkers including: total cholesterol and C-reactive protein (CRP) [27]. Standardised instruments assessed anthropometric measurements including: weight and height. Body mass index (BMI) was calculated by dividing weight $(\mathrm{kg})$ by height $\mathrm{t}^{2}\left(\mathrm{~m}^{2}\right)$. Systolic and diastolic blood pressure (BP) including systolic and diastolic were measured using an automatic device (Spengler SP9).

Rescreening (2001-2004) 
Men self-completed the same standardised questionnaires as in 1991-1994 [27]. The exceptions included: no collection of dietary data or completion of an alcohol diary. Instead, alcohol intake was assessed by asking how many pints of beer, glasses of wine or measures of spirits were consumed within the past three months. Blood samples were analysed for total cholesterol and CRP using the same baseline methods in the QUB laboratory. A clinical examination of weight, height and BP was carried out using the same baseline methods. An oral and dental examination also took place at this phase with 1,558 men from Belfast. This was carried out by one of four dental hygienists to identify if teeth were present and if so how many, while it also identified whether the men had any denture(s) and in which arch (es). They were trained prior to the study to a "gold standard" and inter- and intraexaminer consistency and reproducibility were assessed with regular monthly meetings [29]. Both dentate and edentate men also had a dental radiograph (Orthopantomogram), which confirmed the presence or absence of teeth. In the current study, oral health status was categorised into five groups based on the functional dentition cut-off and prosthodontic rehabilitation [13]: 21-28 teeth with dentures $(n=111), 21-28$ teeth without dentures $(n=453), 1-20$ teeth with dentures $(n=354), 1-20$ teeth without dentures $(n=99)$ and edentate with dentures $(n=79)$.

Follow up (2015)

Diet was assessed using a short food frequency questionnaire (FFQ). This was posted to 1,772 men, of which 1,458 men self-completed and returned via a freepost envelope ( $82.3 \%$ response rate). The FFQ was based on the MD and indicated how often, on average, participants ate specified amounts of food during the past year. There were eight food groups with the portion shown in brackets, including: olive oil/rapeseed oil (1 tablespoon), fruit and natural fruit juice $(80 \mathrm{~g})$, vegetables $(80 \mathrm{~g})$, oily fish $(140 \mathrm{~g})$, wine $(125 \mathrm{ml})$, red meat (1 medium serving), wholegrains (1 medium serving) and nuts (1 small handful). Participants responded with one of the following nine options: never or less than once a month, 1-3/month, once/week, 2-4/week, 5-6/week, once a day, 2-3/day, 4-5/day and 6+/day.

Dietary intake was converted to daily portions and used to generate two diet quality scores: Mediterranean Diet Score (MDS) and the Dietary Diversity Score (DDS). The MDS was used to assess adherence to the MD using the median cut-off values in the PRIME study, as described in Table 1. Protective food components were assigned a value of one when intake was above the cut-off and a zero otherwise, whilst detrimental food groups were assigned a value of one when intake was below the cut-off and a zero otherwise. Individual food group scores were summed together and a higher mean MDS indicated greater adherence to the MD, ranging from 0 to 8 . Diet diversity was also assessed as a measure of diet quality, by assessing the frequency of consumption of the eight main food groups. Participants consuming the food groups at least once a week were assigned a value of one, and a value of zero otherwise. The individual food group scores were summed together to give an overall DDS, ranging from 0 to 8 . A higher mean DDS represented a better dietary diversity and 
variety of foods in the diet. This chosen method was based on a validated 11-item Food Diversity Score used previously in Japan $[22,23]$. These indices were considered the best options, not only because the dietary information was based on food groups, but also because specific nutrient data required for other indices were not calculated in the current study, due to the limited number of foods included in the FFQ.

\section{Statistical Analysis}

All variables were summarised using descriptive statistics and presented according to five oral health groups including: 21-28 teeth with dentures, 21-28 teeth without dentures, 1-20 teeth with dentures, 120 teeth without dentures and edentate with dentures. Means and standard deviations (or standard errors) were presented for continuous variables and frequencies and percentages for categorical variables. Skewed variables (CRP and food daily portions) were log transformed and summarised as geometric means and $95 \%$ confidence intervals. Comparison of continuous variables between groups $(n>2)$ was carried out using ANOVA and for categorical variables $(n>2)$ chi-square tests were used. Associations between oral health status, dietary intakes, dietary recommendations and diet quality were assessed using linear and logistic regression models. Analysis were initially unadjusted and then fully adjusted for potential covariates including: age, socio-economic status (SES), smoking, body mass index (BMI), and C - Reactive Protein (CRP) levels. Statistical analysis was carried out using SPSS for Windows version 21.0. The level of statistical significance was set at $\mathrm{P}<0.05$.

\section{$\underline{\text { Results }}$}

Baseline characteristics for the five oral health status groups are shown in Table 2. Compared to the other four oral health groups, edentate men with dentures were significantly older, had a higher BMI and higher CRP levels. Men with 1-20 teeth with and without dentures had a significantly higher BMI, compared to men with 21-28 teeth with and without dentures. The oral health characteristics of the study population are also shown in Table 2. Overall, the mean number of teeth was 18.5 and ranged from 0 to 28 teeth. Of this, $51.5 \%(n=564)$ had less than 21 natural teeth and $48.5 \%(n=532)$ had 21 or more natural teeth. In terms of denture use, $49.6 \%(n=544)$ had dentures, which consisted of $30.7 \%(n=336)$ with upper dentures, 3.6\% $(n=39)$ with lower dentures and $15.1 \%(n=166)$ with both sets of dentures. The mean number of teeth was highest in men with 21-28 teeth without dentures (24.5), followed by 21-28 teeth with dentures (22.8), 1-20 teeth without dentures (17.9) and 1-20 teeth with dentures (13.7). The majority of men with either 1-20 teeth or 21-28 teeth had upper dentures ( $70 \%$ and $80 \%$ respectively), compared to $96 \%$ of edentate men who had both sets of dentures.

Table 3 shows the mean daily food portions according to number of teeth and prosthodontic rehabilitation. After full adjustment, for age, SES, smoking, BMI and CRP levels, there were significant associations between oral health status and intakes of fruit, vegetables, nuts, and wine after 13 years. The mean fruit intake was significantly higher in each oral health group compared to 
edentate men with dentures: $21-28$ teeth without dentures $(\mathrm{P}=0.001), 21-28$ teeth with dentures $(\mathrm{P}=0.002), 1-20$ teeth without dentures $(\mathrm{P}=0.03)$, and 1-20 teeth with dentures $(\mathrm{P}=0.03)$. The mean future dietary intake of vegetables, nuts, and wine were significantly higher in men with 21-28 teeth without dentures and also in men with 21-28 teeth with dentures, each compared to men with 1-20 teeth without dentures (all $\mathrm{P}<0.01$ ), 1-20 teeth with dentures (all $\mathrm{P}<0.05$ ) and edentate with dentures (both $\mathrm{P}<0.05$ and $\mathrm{P}<0.01$, respectively). The overall $\mathrm{p}$ values for wholegrains and oily fish were not significant, however in the post-hoc analysis, the mean intake was significantly higher in men with 21-28 teeth with dentures, compared to men with 1-20 teeth without dentures (both $\mathrm{P}=0.02$ ). The mean intake of wholegrains was also significantly higher in men with 21-28 teeth without dentures, compared to men with 1-20 teeth without dentures $(\mathrm{P}=0.03)$. The future mean dietary intake of olive oil/rapeseed oil and red and processed meat were not associated with oral health status after full adjustment. Within this analysis, a significant $\mathrm{p}$ for trend across oral health categories was observed for the dietary intake of certain food groups (i.e. fruit, vegetables, and nuts).

Table 4 shows the odds of the impact of number of teeth and prosthodontic rehabilitation on the achievement of UK dietary recommendations after 13 years. In the total sample, $95 \%$ of men were meeting the wine guideline, $31 \%$ oily fish, $27 \%$ fruit and only $3 \%$ for vegetables. In fully adjusted models, edentate men with dentures were $64 \%$ less likely to achieve the target intake of fruit [OR 0.36(95\% CI: 0.17, 0.79; $\mathrm{P}=0.01$ ], compared to men with 21-28 teeth without dentures. Achievement of the other dietary recommendations (i.e. vegetables, oily fish and wine) were not associated with oral health status. Table 5 shows the mean diet quality scores according to number of teeth and prosthodontic rehabilitation. After full adjustment, the mean DDS and MDS were significantly higher in men with 21-28 teeth without dentures, compared to men with 1-20 teeth without dentures (all $\mathrm{P}<0.01$ ), 1-20 teeth with dentures (all $\mathrm{P} \leq 0.001$ ), and edentate with dentures (all $\mathrm{P}<0.001$ ). Similarly, after full adjustment, the mean future DDS and MDS were significantly higher in men with 21-28 teeth with dentures, compared to men with 1-20 teeth without dentures (all $\mathrm{P}<0.05$ ) 1-20 teeth with dentures (all $\mathrm{P}<0.05$ ) and edentate with dentures (all $\mathrm{P}<0.01$ ). Within this analysis, a significant $\mathrm{p}$ for trend across oral health categories was observed for the mean diet quality scores (DDS and MDS).

\section{Discussion}

This study investigated the association between oral health status and both the future dietary intake and diet quality, after an average time period of 13 years in community-dwelling older adults. This is the first study to explore this association between oral health status and adherence to the MD. A significant finding of this study was that an individual's oral health status was associated with future dietary behaviour. Of interest, having 21 or more natural teeth, was positively associated with intakes of fruit, vegetables, and nuts, and higher diet quality scores, compared to the reduced dietary intake and diet quality scores in those with 1-20 teeth or no natural teeth. Therefore, a greater number of 
natural teeth may make foods easier to bite and chew, while having less teeth may make hard, crunchy texture foods uncomfortable and difficult to eat. This may result in the avoidance of particular foods, and the possibility that softer, more energy dense foods are chosen, which increases the intake of saturated fat and sugar [30]. However, the adoption of different food preparation strategies common in the MD can still mean that nutrients can be obtained with a dental impairment (i.e. less teeth or removable dentures), such as softening foods by cooking them for longer [31]. Another option is that fruit juice could be chosen instead of hard fruits [32]. However, cooking methods were not assessed in the current study.

This finding from the current study supports the majority of longitudinal studies, which found an association between tooth loss and reduced future dietary intake [26]. Findings also supported crosssectional studies in older adults in the UK, US and Asian populations, as those with less natural teeth had a reduced consumption of fruit and vegetables $[16,19,33]$, while in the US there were reduced intakes of meat, beans and oils and increased intakes of fat, alcohol and added sugar, compared to those with a greater number of natural teeth [19]. However, in the current study, meat and oils were not significantly associated with oral health status, whilst wine intake was higher in men with a greater number of teeth. Differences may be explained by the use of non-specific types of meat, lack of alcohol categories and a larger edentate sample size $(n=221)$ in that analysis [19], compared to the current study $(\mathrm{n}=79)$. Similar to the current study, having 21 or more natural teeth was associated with a greater consumption of fruit and vegetables, and subsequently fibre intake [34]. Therefore, 21 or more natural teeth may help achieve optimum nutritional intake [35]. To date no studies have prospectively investigated whether oral health has an impact on diet quality several years later. The current study findings support evidence which found that less natural teeth was associated with a lower DDS in older females in Japan [23], and also in adults aged over 50 years old in India with $\geq 10$ teeth missing/decayed [24]. However, this association did not exist in males [23], which is not in agreement with the current study findings. Similarly, the current study findings do not support evidence which suggested that 21 or more natural teeth was not associated with a higher diet quality score in over 60 year olds in the US [25]. This difference may be due to the different diet quality scores used, as that analysis used the Healthy Eating Index [25], while the current study included only men, had a different sample size and categorisation of oral health status groups and food groups.

Interestingly, the current study results suggest that the effect of severe tooth loss on the future dietary intake and diet quality may not be overcome with removable dentures, as edentate men with dentures had the lowest intake of fruit and vegetables, whilst men with 1-20 teeth with dentures had the lowest intake of nuts. Edentate men with dentures also had the lowest diet quality scores and were less likely to achieve the fruit dietary recommendation of two portions per day in the future. Results suggest that the use of dentures in those with less than 21 teeth was not associated with the same level of future dietary intake of fruit, vegetables, and nuts, as those with 21 or more teeth with dentures. However, it 
is important to acknowledge that this was a broad category, as it included for example, someone with two natural teeth and 18 artificial teeth, as well as someone with five natural teeth and five artificial teeth. Food choice and ability to eat certain hard-textured foods may differ between participants within this oral health status category. Therefore, it is not clear whether the reduced intake of fruit, vegetables and nuts was associated with the use of dentures or having less natural teeth.

Findings in the current study do, however, support findings of the lower consumption of fruit and vegetables in older adults with complete dentures in Finland [36]. The use of complete dentures was also associated with a reduced nutrient intake in older adults in the UK [37], and particularly, energy, carbohydrates, lipids and fibre in France [38] compared to fully dentate older adults. Older adults in Japan with less than $\leq 5$ occluding pairs of natural teeth (OPNT) and self-perceived ill-fitting dentures was associated with a lower DDS [22]. While the current study did not investigate nutrient intake, it is assumed that reduced dietary intake may be linked with a reduced nutrient intake. The current study supports findings in the US, as severe tooth loss was associated with being less likely to achieve various dietary guidelines (e.g. total fruit, whole fruit, total vegetables, meat and beans, and oils), compared to moderate to low tooth loss [19]. A combination of prosthodontic rehabilitation with dietary guidance may be required to improve dietary intake [39], and this proved successful in increasing fruit and vegetable consumption in edentate individuals [18]. Therefore, having a better oral health status alone, is not enough to change dietary intake and improve diet quality. However, it was not clear if those with severe tooth loss wore dentures [19], if dentures were kept in during food consumption or if they fitted correctly in these studies $[36,38,39]$.

The current study does, however, lend support to the use of dentures for better nutritional intake, at least in those with 21 or more natural remaining teeth. Partially dentate men with 21-28 teeth with dentures had a higher intake of fruit, vegetables, and nuts, and diet quality scores, compared to those with 1-20 teeth with dentures. Having 21 or more natural remaining teeth may play a supportive role during the use of dentures, and subsequently, aid future dietary intake and diet quality, such as enabling men to consume more food groups in the MD. The finding supports recent evidence in Japan, where removable partial dentures were associated with an improvement in dietary intake, but only in those who had lost a small number of teeth [40].

\section{Strengths and Limitations}

The strengths of this study included the relatively large representative sample of older Northern Irish men $(n=1,096)$. Results may be generalizable to other community-dwelling male populations with similar oral health status. Oral health status was assessed by one of four dental hygienists, trained to a gold standard, and inter- and intra-examiner consistency and reproducibility were assessed monthly. Dietary intake over the previous year was assessed using a short FFQ, by posting it to participants' homes. This helped to reduce social desirability bias, as participants were not completing the 
questionnaire with the researcher. A relatively high response rate $(82.3 \%)$ was achieved for the completion of the FFQ. The FFQ was based on MD food groups adapted for a NI population, to ensure that they were foods likely to be consumed in NI.

However, a number of limitations must be acknowledged. As male community-dwelling older adults were recruited, findings are not generalizable to older females or those living in residential or care homes. This is important as oral health status and diet can vary between genders $[13,41]$ and edentulism is higher in these facilities compared to private households [42]. Further research is required in prospective studies with larger populations, different genders, and more detailed dietary assessment. Specifically, prosthodontic rehabilitation in those with 21 or more natural remaining teeth and future dietary intake and diet quality. It was not clear if dentures were kept in or removed during food consumption, and the denture quality and fit were unknown. The number of natural teeth and artificial teeth (i.e. dentures) in the categories was broad and this may have affected food choices, and therefore this should be explored further. Other potential confounding factors (i.e. energy intake, health knowledge, cooking skills or food preferences) not investigated in this current study, may have led to different findings if they were included in the analysis. The edentate with dentures group had a smaller sample size $(n=79)$ and this may have led to a reduced power to detect results as statistically significant, and the use of multiple comparisons may have resulted in significant findings due to chance. The FFQ did not provide a true representation of participant's overall diet as the men will have consumed other foods. There was a risk of under- or over-reporting of dietary intake, which may be due to recall bias that can be prevalent in this age group. Average intakes may have underestimated achieving the vegetables dietary guideline. Some participants did not answer all eight questions in the FFQ, so either the food group was not truly consumed or it was a missing response. It was not clear if oral health status had an impact on the consumption of individual fruits or vegetables as the categories were generic. Dietary intake may have changed after the oral health assessment, or upon receiving dentures, but this was not recorded in 2001-2004. Objective biochemical measures would have been useful to confirm dietary intake.

\section{Conclusions}

Oral health status, specifically the number of remaining natural teeth and prosthodontic rehabilitation, was associated with dietary intake and diet quality in older men, after an average time period of 13 years. Having 21 or more natural teeth remaining positively affected the future dietary intake of fruit, vegetables, and nuts, and resulted in higher diet quality scores (MDS and DDS), compared to those with severe tooth loss (i.e. 1-20 teeth or edentate). Therefore, the findings support the view that it is important to retain as many natural teeth as possible in later life. Men who were edentate and wearing dentures had the lowest diet quality scores and were less likely to achieve recommendation for daily fruit intake. Prosthodontic rehabilitation may be beneficial for dietary intake and diet quality, 
especially, in those with at least 21 or more natural remaining teeth, in terms of improving the intake of hard-texture foods such as fruits, vegetables and nuts. Overall, findings suggest that oral health status is positively associated with future dietary intake, with those with a larger number of natural remaining teeth achieving better quality dietary intake.

\section{Declaration of interest: None}

Funding: The PRIME study was originally funded by a Programme grant from the NI HSC Research and Development Fund and the British Heart Foundation. The PhD studentship was funded by the Department for the Economy in Northern Ireland.

\section{$\underline{\text { References }}$}

[1] United Nations, World population Ageing, 2015. doi:10.5860/CHOICE.40-1307.

[2] M.J. Prince, F. Wu, Y. Guo, L.M. Gutierrez Robledo, M. O’Donnell, R. Sullivan, S. Yusuf, The burden of disease in older people and implications for health policy and practice, Lancet. 385 (2015) 549-562. doi:10.1016/S0140-6736(14)61347-7.

[3] R. Estruch, E. Ros, J. Salas-Salvadó, M.-I. Covas, D. Corella, F. Arós, E. Gómez-Gracia, V. Ruiz-Gutiérrez, M. Fiol, J. Lapetra, R.M. Lamuela-Raventos, L. Serra-Majem, X. Pintó, J. Basora, M.A. Muñoz, J. V. Sorlí, J.A. Martínez, M. Fitó, A. Gea, M.A. Hernán, M.A. Martínez-González, Primary prevention of cardiovascular disease with a mediterranean diet supplemented with extra-virgin olive oil or nuts, N. Engl. J. Med. 378 (2018) e34. doi:10.1056/NEJMoa1800389.

[4] National Health Service, What is a Mediterranean diet? -, (2017). https://www.nhs.uk/livewell/eat-well/what-is-a-mediterranean-diet/ (accessed October 25, 2018).

[5] World Health Organisation, Food based dietary guidelines in the WHO European Region, 2003. http://www.euro.who.int.

[6] SACN, Iron and Health, 2010.

[7] SACN, Advice on fish consumption: benefits \& risks 2004, 2004. doi:0 11243083 X.

[8] A. Alkerwi, Diet quality concept, Nutrition. 30 (2014) 613-618. doi:10.1016/j.nut.2013.10.001.

[9] R. Micha, S. Khatibzadeh, P. Shi, K.G. Andrews, R.E. Engell, D. Mozaffarian, Global, regional and national consumption of major food groups in 1990 and 2010: A systematic analysis including 266 country-specific nutrition surveys worldwide, BMJ Open. 5 (2015) 123. doi:10.1136/bmjopen-2015-008705. 
[10] A. Host, A.T. McMahon, K. Walton, K. Charlton, Factors influencing food choice for independently living older people — a systematic literature review, J. Nutr. Gerontol. Geriatr. 35 (2016) 67-94. doi:10.1080/21551197.2016.1168760.

[11] B.A. Dye, S. Tan, V. Smith, B.G. Lewis, L.K. Barker, G. Thornton-Evans, P.I. Eke, E.D. Beltra'n-Aguilar, A.M. Horowitz, Trends in oral health status: United States, 1988 - 1994 and $1999-2004,2007$.

[12] Centers for Disease Control and Prevention, Oral health surveillance report: Trends in dental caries and sealants, tooth retention, and edentulism, United States 1999-2004 to 2011-2016, Atlanta, GA, 2019.

[13] E. Fuller, J. Steele, W. Watt, N. Nuttall, 1: Oral health and function - a report from the Adult Dental Health Survey 2009, 2011. http://www.hscic.gov.uk/catalogue/PUB01086/adul-dentheal-surv-summ-them-the1-2009-rep3.pdf.

[14] N.J. Kassebaum, E. Bernabé, M. Dahiya, B. Bhandari, C.J.L. Murray, W. Marcenes, Global burden of severe tooth loss, J. Dent. Res. 93 (2014) 20S-28S. doi:10.1177/0022034514537828.

[15] C.F. Driscoll, M.A. Freilich, A.D. Guckes, K.L. Knoernschild, T.J. Mcgarry, G. Goldstein, C. Goodacre, A. Guckes, S. Mor-, S. Rosenstiel, C. Vanblarcom, The Glossary of Prosthodontic Terms, J. Prosthet. Dent. 117 (2017) C1-e105. doi:10.1016/j.prosdent.2016.12.001.

[16] Q. Zhang, D. Niesten, E.M. Bronkhorst, D.J. Witter, N.H.. Creugers, Food avoidance is associated with reduced dentitions and edentulousness, Clin. Oral Investig. (2019) 1-8.

[17] M.P. Toniazzo, P. de S. Amorim, F.W.M.G. Muniz, P. Weidlich, Relationship of nutritional status and oral health in elderly: Systematic review with meta-analysis, Clin. Nutr. (2017) 1-7. doi:10.1016/j.clnu.2017.03.014.

[18] A. Tada, H. Miura, Systematic review of the association of mastication with food and nutrient intake in the independent elderly, Arch. Gerontol. Geriatr. 59 (2014) 497-505. doi:10.1016/j.archger.2014.08.005.

[19] M. Savoca, T. Arcury, X. Leng, H. Chen, R. Bell, A. Anderson, T. Kohrman, R. Frazier, G. Gilbert, S. Quandt, Severe tooth loss in older adults as a key indicator of compromised dietary quality., Public Health Nutr. 13 (2010) 466-474. doi:https://dx.doi.org/10.1017/S1368980009991236.

[20] M.R. Savoca, T.A. Arcury, X. Leng, H. Chen, R.A. Bell, A.M. Anderson, T. Kohrman, G.H. Gilbert, S.A. Quandt, Impact of denture usage patterns on dietary quality and food avoidance among older adults., J. Nutr. Gerontol. Geriatr. 30 (2011) 86-102. 
doi:https://dx.doi.org/10.1080/01639366.2011.545043.

[21] Y. Zhu, J.H. Hollis, Tooth loss and its association with dietary intake and diet quality in American adults, J. Dent. 42 (2014) 1428-1435. doi:10.1016/j.jdent.2014.08.012.

[22] M. Iwasaki, Y. Kimura, A. Yoshihara, H. Ogawa, T. Yamaga, T. Wada, R. Sakamoto, Y. Ishimoto, E. Fukutomi, W. Chen, M. Fujisawa, K. Okumiya, M.C. Manz, T. Ansai, Low dietary diversity among older Japanese adults with impaired dentition, J. Dent. Oral Hyg. 7 (2015) 71-77. doi:10.5897/JDOH2015.0147.

[23] M. Iwasaki, Y. Kimura, A. Yoshihara, H. Ogawa, T. Yamaga, T. Takiguchi, T. Wada, R. Sakamoto, Y. Ishimoto, E. Fukutomi, W. Chen, H. Imai, M. Fujisawa, K. Okumiya, M.C. Manz, H. Miyazaki, K. Matsubayashi, Association between dental status and food diversity among older Japanese, Community Dent. Health. 32 (2015) 104-110. doi:10.1922/CDH.

[24] B. Agarwal, N. Kumari, S. Shukla, S. Shukla, T. Shahi, A. Asif, Dietary diversity and nutritional status : A relationship with loss of functional teeth, J. Physiol. Anthropol. 13 (2014) $41-46$

[25] R.B. Ervin, B.A. Dye, The effect of functional dentition on healthy eating index scores and nutrient intakes in a nationally representative sample of older adults, Am. Assoc. Public Heal. Dent. 69 (2009) 207-216. doi:10.1111/j.1752-7325.2009.00124.x.

[26] P. Gaewkhiew, W. Sabbah, E. Bernabé, Does tooth loss affect dietary intake and nutritional status? A systematic review of longitudinal studies, J. Dent. 67 (2017) 1-8. doi:10.1016/j.jdent.2017.10.012.

[27] J. Yarnell, The PRIME study: classical risk factors do not explain the severalfold differences in risk of coronary heart disease, Q J Med. 91 (1998) 667-676.

[28] A. Wagner, C. Simon, A. Evans, P. Ducimetiere, V. Bongard, M. Montaye, D. Arveiler, G. PS, Physical activity patterns in 50-59 year men in France and Northern Ireland. Associations with socio-economic status and health behaviour, Eur J Epidemiol. 18 (2003) 321-329.

[29] G.J. Linden, K.M. McClean, J. V. Woodside, C.C. Patterson, A. Evans, I.S. Young, F. Kee, Antioxidants and periodontitis in 60-70-year-old men, J. Clin. Periodontol. 36 (2009) 843-849. doi:10.1111/j.1600-051X.2009.01468.x.

[30] M.R. Savoca, T.A. Arcury, X. Leng, H. Chen, R.A. Bell, A.M. Anderson, T. Kohrman, G.H. Gilbert, S.A. Quandt, Food avoidance and food modification practices due to oral health problems linked to the dietary quality of older adults, J. Am. Geriatr. Soc. 58 (2010) 12251232. doi:10.1111/j.1532-5415.2010.02909.x. 
[31] A. Kossioni, O. Bellou, Eating habits in older people in Greece: The role of age, dental status and chewing difficulties, Arch. Gerontol. Geriatr. 52 (2011) 197-201.

doi:10.1016/j.archger.2010.03.017.

[32] P. Moynihan, M. Thomason, A. Walls, K. Gray-Donald, J.A. Morais, H. Ghanem, S. Wollin, J. Ellis, J. Steele, J. Lund, J. Feine, Researching the impact of oral health on diet and nutritional status: methodological issues., J. Dent. 37 (2009) 237-249.

doi:https://dx.doi.org/10.1016/j.jdent.2008.12.003.

[33] A. Sheiham, J. Steele, Does the condition of the mouth and teeth affect the ability to eat certain foods, nutrient and dietary intake and nutritional status amongst older people?, Public Health Nutr. 4 (2001) 797-803. doi:10.1079/PHN2000116.

[34] W. Marcenes, J.G. Steele, A. Sheiham, A.W.G. Walls, The relationship between dental status, food selection, nutrient intake, nutritional status, and body mass index in older people., Cad. Saude Publica. 19 (2003) 809-816.

http://ovidsp.ovid.com/ovidweb.cgi?T=JS\&PAGE=reference $\& D=$ med4\&NEWS=N\&AN=128 06483.

[35] A. Sheiham, J.G. Steele, W. Marcenes, C. Lowe, S. Finch, C.J. Bates, A. Prentice, A.W. Walls, The relationship among dental status, nutrient intake, and nutritional status in older people., J. Dent. Res. 80 (2001) 408-413. doi:https://dx.doi.org/10.1177/00220345010800020201.

[36] L. Jauhiainen, S. Mannisto, P. Ylostalo, M. Vehkalahti, A. Nordblad, A.W. Turunen, A.L.N. Suominen, Food consumption and nutrient intake in relation to denture use in 55- to 84-yearold men and women - results of a population based survey., J. Nutr. Health Aging. 21 (2017) 492-500. doi:https://dx.doi.org/10.1007/s12603-016-0793-z.

[37] S. Watson, C.R. Cardwell, J.V. Woodside, G. McKenna, The impact of dental status on perceived ability to eat certain foods, nutrient intake and nutritional status in older adults: UK National Diet and Nutrition Survey 2008-2012, Int. J. Behav. Nutr. Phys. Act. 16 (2019) 1-13. doi:10.1017/s0029665117001835.

[38] P.Y. Cousson, M. Bessadet, E. Nicolas, J.-L. Veyrune, B. Lesourd, C. Lassauzay, Nutritional status, dietary intake and oral quality of life in elderly complete denture wearers., Gerodontology. 29 (2012) e685-92. doi:https://dx.doi.org/10.1111/j.1741-2358.2011.00545.x.

[39] R.B. Ervin, B.A. Dye, Number of natural and prosthetic teeth impact nutrient intakes of older adults in the United States., Gerodontology. 29 (2012) e693-702. doi:https://dx.doi.org/10.1111/j.1741-2358.2011.00546.x. 
[40] C. Inomata, K. Ikebe, T. Okada, H. Takeshita, Y. Maeda, Impact on dietary intake of removable partial dentures replacing a small number of teeth, Int. J. Prosthodont. 28 (2015) 583-585. doi:https://dx.doi.org/10.11607/ijp.4306.

[41] J. Wardle, D. Ph, A.M. Haase, A. Steptoe, D. Phil, A.B. Med, Gender differences in food choice: the contribution of health beliefs and dieting, Ann. Behav. Med. 27 (2004) 107-116.

[42] M. Morgan, W. Oral Health, J. Neville, E. Rooney, What is known about the oral health of older people in England and Wales: A review of oral health surveys of older people, 2015. www.gov.uk/phe\% $\% 5$ Cnwww.gov.uk/phe. 